\title{
3D Finite Element Model of Aqueous Outflow to Predict the Effect of Femtosecond Laser Created Partial Thickness Drainage Channels
}

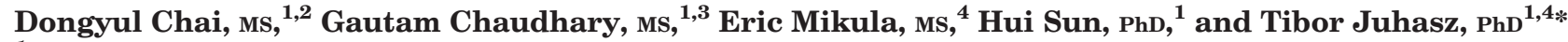 \\ ${ }^{1}$ Department of Ophthalmology, University of California-Irvine, Irvine, California 92697 \\ ${ }^{2}$ Department of Biomedical Engineering, University of Michigan, Ann Arbor, Michigan 48109 \\ ${ }^{3}$ Department of Electrical Engineering and Computer Science, University of California-Irvine, Irvine, California 92697 \\ ${ }^{4}$ Department of Biomedical Engineering, University of California-Irvine, Irvine, California 92697
}

Background and Objectives: Partial thickness drainage channels can be created with femtosecond lasers in the translucent sclera for the potential treatment of glaucoma. We present a 3D finite element model (FEM) that can predict the effect of these channels on aqueous humor $(\mathrm{AH})$ outflow and intraocular pressure (IOP).

Study Design/Materials and Methods: A 3D model was developed based on a 2D model for the intact eye using COMSOL (Comsol, Inc., MA) finite element software. Different values of permeability were entered into the $3 \mathrm{D}$ model for the AH pathway and for the partial thickness channel. To obtain experimental data for model validation, one partial thickness channel was created in each of three enucleated rabbit eyes with a femtosecond laser tuned to $1.7 \mu \mathrm{m}$ wavelength. Aqueous outflow rates were measured with the perfusion method before and after the laser treatments at different levels of IOP and then compared to IOP values predicted by the model.

Results: The experiments indicated that the rate of the $\mathrm{AH}$ outflow was increased in each of three eyes after the laser treatment. Assuming a constant rate of $\mathrm{AH}$ production the $3 \mathrm{D}$ model predicted IOP reductions ranging from $67.2 \%$ to $80.6 \%$ as the effect of the laser created channels. These predictions were in reasonable agreement with experimentally adjusted IOP values during the perfusion measurements.

Conclusions: The developed 3D FEM has the potential to predict IOP reduction caused by partial thickness drainage channels created with the femtosecond laser in the sclera. Such a model may also be used to determine optimal channel dimensions for a specified increase in outflow facility and reduction in IOP. Lasers Surg. Med. 40:188195, 2008. (c) 2008 Wiley-Liss, Inc.

Key words: glaucoma; aqueous humor; intraocular pressure

\section{INTRODUCTION}

Open angle glaucoma is progressive optic neuropathy that may be associated with increased intra-ocular pressure. It is believed that there exists compressive damage to retina ganglion cells at the lamina cribrosa in the lamina optic nerve head and it may be a significant factor in the pathophysiology of this disease [1,2]. Elevated IOP is primarily due to a reduction in aqueous humor (AH) flow rate through outflow pathway [2]. Current glaucoma treatments include surgical and drug treatments to both decrease $\mathrm{AH}$ formation and to increase $\mathrm{AH}$ outflow facility. Surgical treatments offer potential advantages over drug therapies, including generally larger IOP reductions and reduced patient compliance requirements [3]. However, current incisional and laser surgical procedures also have significant disadvantages such as perioperative complications and limited longevity of achieved IOP reductions [4]. In addition, surgical treatments may be associated with increased risk of intra-ocular infection. Femtosecond laser pulses can achieve laser induced optical breakdown (LIOB) at relatively low pulse energies [5-7] and can be delivered beneath the translucent sclera by selecting the proper wavelength and scanning patterns [8]. Our previous studies have demonstrated that such subsurface scleral channels can be created in cadaver eyes with $1.7 \mu \mathrm{m}$ femtosecond laser pulses and that these channels reduce IOP by increasing outflow rates [9]. Since the ultimate goal of such a glaucoma treatment is to predictably reduce IOP to a desired range, we investigated quantitative modeling of such surgical treatments. Although, there have been a number of efforts to model AH dynamics [10-12], most have focused on qualitative aspects of $\mathrm{AH}$ flow patterns [11] or the relationship between IOP and changes in ocular structures [10,12]. Most have also relied on 2D models, without comparison to experimental data. In contrast to these studies, we constructed a 3D mathematical model for $\mathrm{AH}$ flow in the rabbit eye and then compared its predictions with experimental data from our ex vivo setup developed in

Contract grant sponsor: National Institutes of Health (NIH); Contract grant number: R01 EY014456-02.

*Correspondence to: Tibor Juhasz, Department of Opthamology, University of California-Irvine, 118 Med Surge I, Irvine, CA 92697.

Accepted 11 December 2007

Published online in Wiley InterScience

(www.interscience.wiley.com).

DOI 10.1002/lsm.20608 
previous studies [13]. Our goal was to investigate the feasibility of predicting IOP with a 3D mathematical model and its potential use as a tool in predetermining the dimensions of the partial thickness channel to reduce IOP predictably in glaucoma management.

\section{MATERIALS AND METHODS}

\section{Finite Element Model}

Basic assumptions. A finite element model (FEM) was developed to simulate the $\mathrm{AH}$ flow dynamics in rabbit eyes based on the following assumptions:

(1) The amount of $\mathrm{AH}$ flowing out of the rabbit eye through the uveoscleral pathway is not exactly known. Therefore, it is assumed that all the $\mathrm{AH}$ outflow occurs through the trabecular pathway.

(2) The trabecular pathway in the rabbit eye is porous, so that outflow through the pathway follows the Brinkman equation and its resistance to outflow can be characterized with permeability in the equation. This assumption is in line with the efforts to quantify the outflow resistance [11], and ultimately to provide regulated decrease in IOP.

(3) The resistance of pathway is characterized with one comprehensive permeability value rather than a summation of the permeability of each component of the pathway.

(4) The structures of the eye such as the cornea are rigid materials, and it is assumed that all structural components such as lens and iris inside the eye do not move with changes in IOP. The relationship between IOP and movement of eye structures has been investigated [10], but it is not the focus of the current study.

(5) The potential impact of temperature and circulatory perfusion on the $\mathrm{AH}$ outflow was not considered since cadaver eyes were used in this study. It was assumed that the fluid flowing into the eye from the reservoir as well as the eye is equilibrated to room temperature. Previous studies demonstrated [11] that there are two potential heat sources that can have impact on $\mathrm{AH}$ flow, the temperature difference between the cornea and the rest of the eye and temperature differences created by the blood flow. These temperature gradients can affect $\mathrm{AH}$ flow, but are neglected in the current model.

Mathematical model. A schematic of the rabbit eye is displayed in Figure 1. Our 2D model was constructed so that it focused on $\mathrm{AH}$ flow dynamics in the anterior chamber. It was shown that the gap between the lens and iris is on the order of micrometers $[12,15]$. This very narrow gap is difficult to mesh and even if it is meshed, it cannot be calculated with reliable convergence and stability, especially in the 3D model without use of a supercomputer. Therefore, our model focused on $\mathrm{AH}$ dynamics in the anterior chamber. The anterior chamber was modeled as a hemisphere with a diameter of $12 \mathrm{~mm}$ along the edge of the iris [11]. The height of the trabecular meshwork (TM) in human eyes ranges from 0.38 to $0.86 \mathrm{~mm}$ [16]. Therefore, the TM in rabbit eye was assumed to have a height of $0.3 \mathrm{~mm}$, lower than human eye. In order to simplify the model, the $\mathrm{AH}$ inflow was assumed to enter anterior chamber through the velocity inlet marked with a red line in Figure 2b. Boundary conditions at velocity inlet were given so that the inflow rate was the same as the one measured in the experiment. This eliminates the problems from meshing the narrow iris-lens gap (see Fig. 2a,b). Similar simplification approaches to model $\mathrm{AH}$ in the anterior chamber has been qualitatively tried [11].

In the living rabbit eye, $\mathrm{AH}$ flows into episcleral vein to exit the eye. Therefore, the final destination of $\mathrm{AH}$ flow should be modeled to be the constant pressure boundary condition, $9.6 \mathrm{mmHg}$, the pressure in the episcleral vein [17]. However, there is no available blood pressure in the

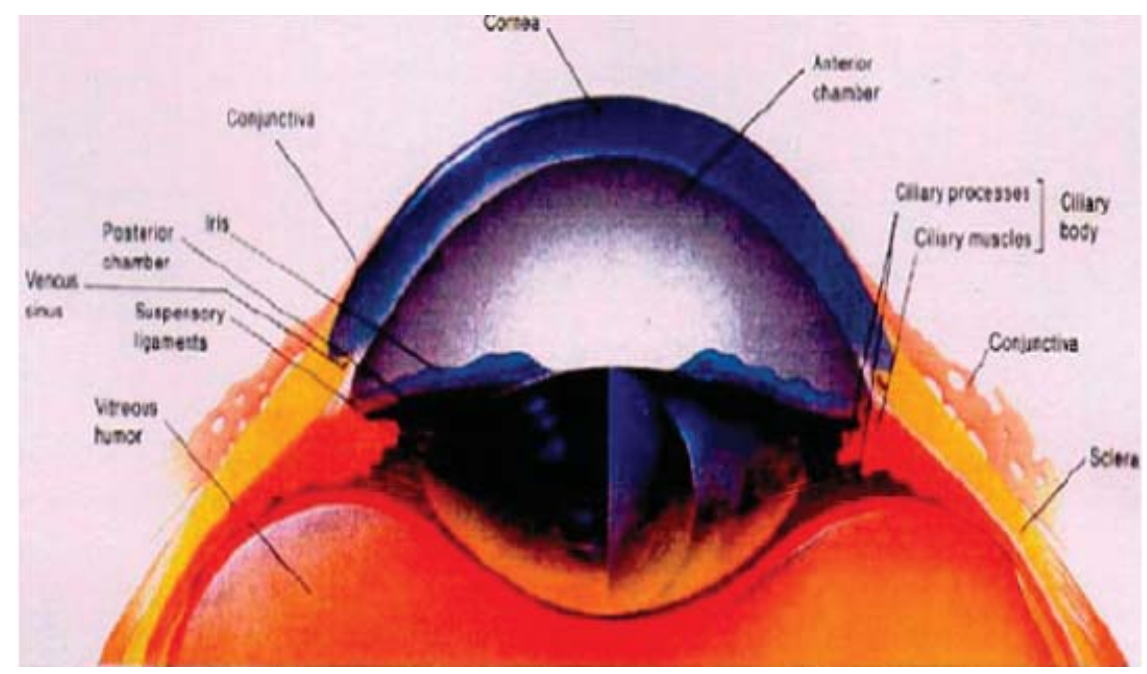

Fig. 1. Schematic of rabbit eye [9]. 

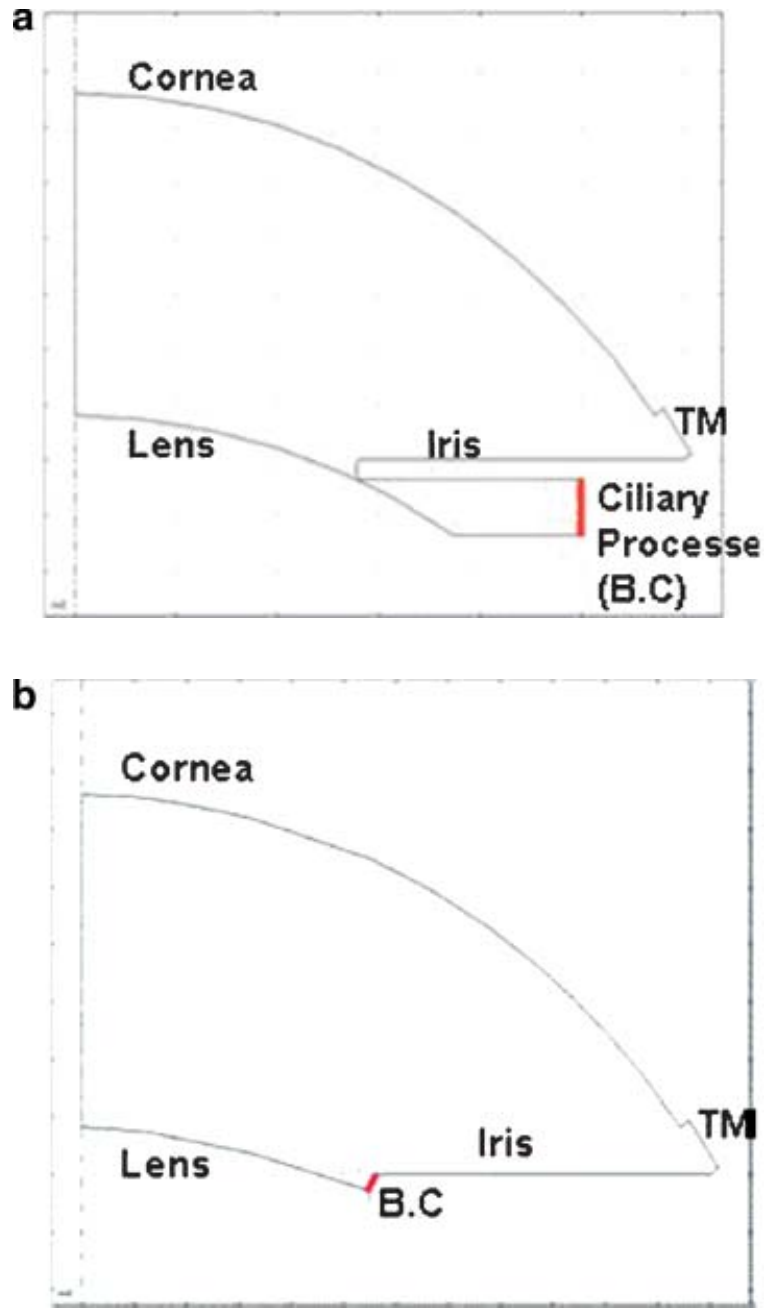

Fig. 2. a: Schematic of outflow pathway used in 2D model. b: Modified 2D model.

episcleral vein in cadaver eyes. Therefore, it is assumed that $\mathrm{AH}$ flows through sclera and the final destination is modeled to be constant boundary condition at the atmospheric pressure. The $3 \mathrm{D}$ model was developed by the revolution of the modified axially symmetric $2 \mathrm{D}$ model by $360^{\circ}$. The geometry of 3D model with the femtosecond laser created channel (marked with a circle) is shown in Figure 3.

Navier-Stokes equation and continuity equation for the $\mathrm{AH}$ described as incompressible fluid in Equation (1) were

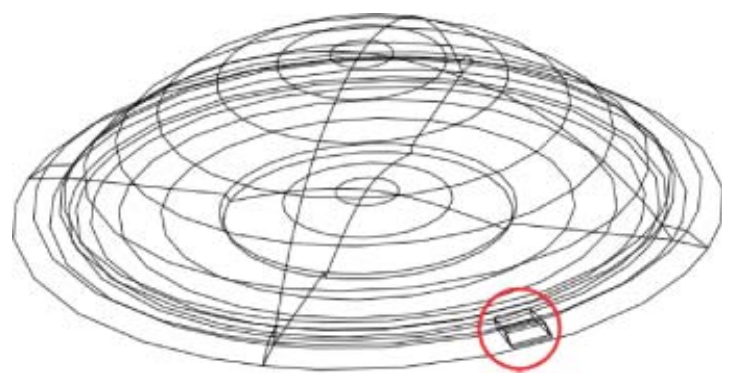

Fig. 3. Geometry of 3D model. modified to Equation (1a) for the steady state flow in the anterior chamber,

$$
\begin{gathered}
\rho \frac{\partial \underline{u}}{\partial t}-\nabla\left[\eta\left(\nabla \underline{u}+(\nabla \underline{u})^{\mathrm{T}}\right)\right]+\rho \underline{u} \nabla \underline{u}+\nabla P=F, \\
\frac{\partial P}{\partial t}+\nabla(\rho \underline{u})=0 \\
-\nabla\left\lfloor\eta\left(\nabla \underline{u}+(\nabla \underline{u})^{\mathrm{T}}\right)\right\rfloor+\rho \underline{u} \nabla \underline{u}+\nabla P=0, \quad \nabla \underline{u}=0
\end{gathered}
$$

Brinkman equation governing the flow through trabecular pathway was modified from Equation (2) to (2a) for steady state flow

$$
\begin{gathered}
\rho \frac{\partial \underline{u}}{\partial t}-\nabla\left[\eta\left(\nabla \underline{u}+(\nabla \underline{u})^{\mathrm{T}}\right)\right]+\nabla P+\frac{\eta}{\kappa} \underline{u}=F, \\
\frac{\partial \rho}{\partial t}+\nabla(\rho \underline{u})=0 \\
-\nabla\left[\eta\left(\nabla \underline{u}+(\nabla \underline{u})^{\mathrm{T}}\right)\right]+\nabla P+\frac{\eta}{\kappa} \underline{u}=0, \quad \nabla \underline{u}=0
\end{gathered}
$$

where $\underline{u}$ is velocity $(\mathrm{m} / \mathrm{s}), \rho$ is density $\left(\mathrm{kg} / \mathrm{m}^{3}\right), \eta$ is dynamic viscosity (Pa second), $F$ is body force, and $\kappa$ is permeability $\left(\mathrm{m}^{2}\right)$. Each equation was modified, because we focused on the steady state flow in anterior chamber and in this case the body force is absent. The properties of $\mathrm{AH}$ used in model were $\rho=1,000 \mathrm{~kg} / \mathrm{m}^{3}, \eta=1.002 \times 10^{-3} \mathrm{~Pa} \cdot \mathrm{s}$. The equations provided above were solved by COMSOL 3.3 (Comsol, Inc., Burlington, MA) for the finite element mesh and boundary conditions described above. The permeability of pathway is calculated by fitting the experimental $\mathrm{AH}$ outflow data measured in untreated enucleated rabbit eyes to the $2 \mathrm{D}$ model.

The channel was created in the 3D model so that the remaining layer between the top of channel and anterior surface of sclera is thin, as described in Figures $4 \mathrm{~b}$ and 9, but it still provides some resistance, characterized with the modified permeability to be referred to as "effective channel permeability," to the outflow.

\section{Experiment}

Experimental setup. Standard perfusion experiments were performed in cadaver rabbit eyes to investigate the effect of drainage channels created by the femtosecond laser, to provide input parameters for the model and compare experimental results to model predictions.

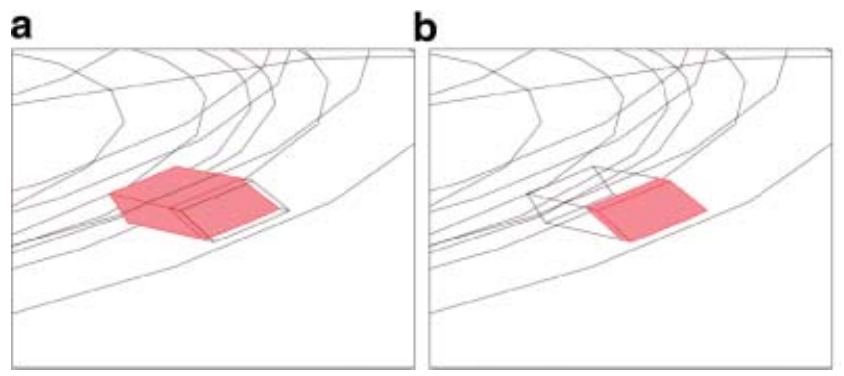

Fig. 4. Geometry used in 3D model showing details of (a) femtosecond laser created partial thickness channel, (b) remaining layer at the anterior surface of sclera. 
Laser and beam delivery system. The Ti:Sapphire laser system was composed of oscillator, pulse stretcher, regenerative amplifier, pulse compressor, and optical parametric amplifier (Coherent, Santa Clara, CA) and arranged as shown in Figure 5. Labview (National Instruments, Austin, TX) was used to develop the software to control the lasers. Amplified pulses with durations less than 130 femtoseconds and pulse energies greater than $0.3 \mathrm{~mJ}$ were produced by the system at approximately $800 \mathrm{~nm}$ wavelength and $5 \mathrm{kHz}$ repetition rate. The pulses from Ti:Sapphire laser system were tuned to $1.7 \mu \mathrm{m}$ wavelength, by an optical parametric amplifier (Coherent). Output laser beam from optical parametric amplifier was expanded through beam expander, reflected by mirrors and focused through 0.5 NA aspheric lens (Thorlabs, Newton, $\mathrm{NJ})$.

Scanning system. A schematic view of the experimental setup to scan the eye is presented in Figure 6. The laser beam was focused into the tissue using a 0.5 NA focusing lens. The enucleated eyes were placed on a three-axis scanner stage driven by three stepper motors, one along each axis (Newport MM1000 and stepper motors; Newport Corp., Irvine, CA). The stepper motors were controlled by a computer using Labview 7.1 (National Instruments) through electronic controller (ESP 300, Newport Corp.). The axis parallel to the propagation of the light (see Fig. 6) is referred to as the $Z$-axis. The $X$-axis is tangential to the corneal perimeter and the $Y$-axis is perpendicular to the $X Z$ plane. Thus, the three scanning axes compose a Cartesian coordinate system. In order to minimize light scattering on the surface of the tissue a microscope slide was placed on the cadaver eye at the proposed location of the channel. The scanner stages were moved along programmed patterns with predetermined velocity to create the partial thickness AH drainage channel in the sclera.

Outflow rate measurements. Figure 7 displays the schematics of the AH outflow measurement setup modified from the one developed by Brubaker [18]. The reservoir

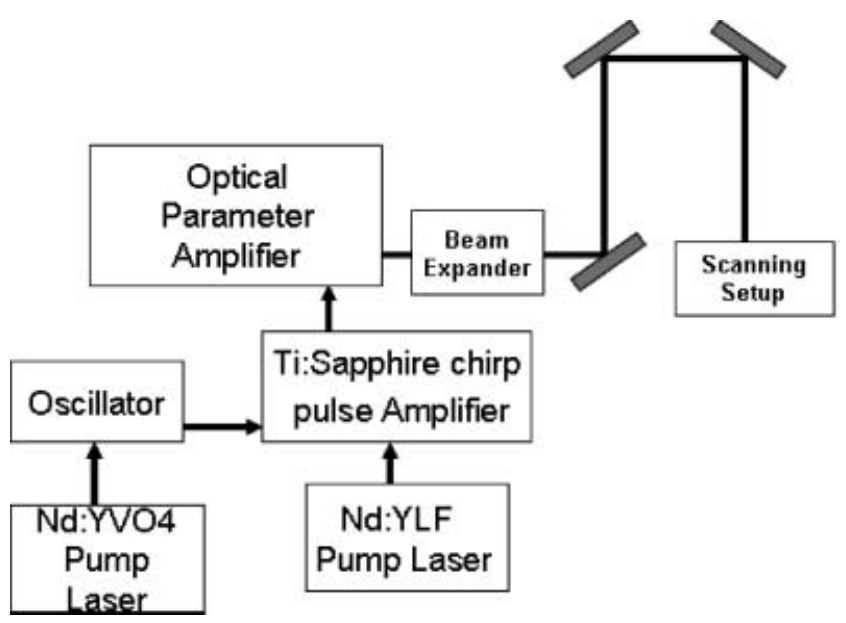

Fig. 5. Schematics of laser and beam delivery optics used in the experiment.

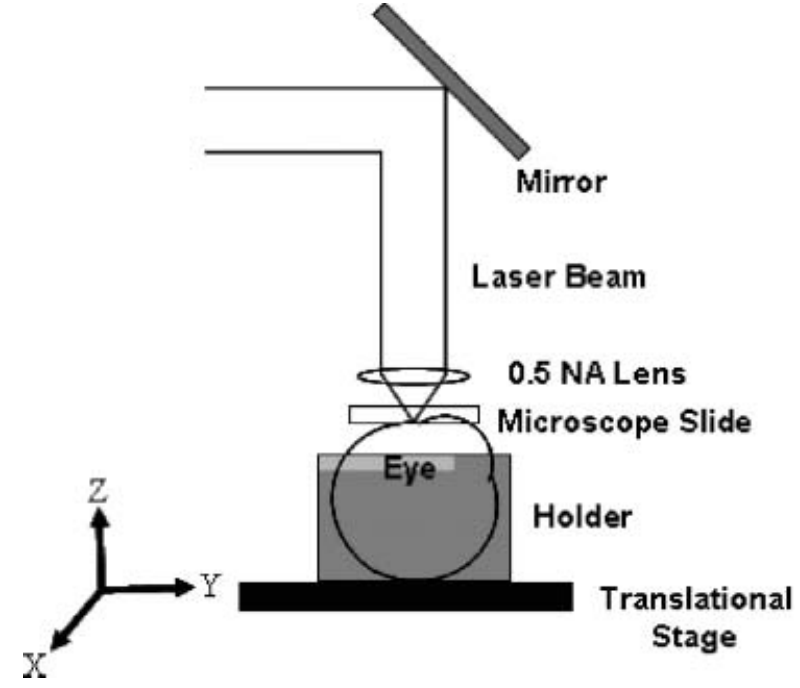

Fig. 6. Schematics of beam scanning mechanism.

containing balanced salt solution (BSS) was connected to the enucleated rabbit eye. A 23-gauge needle with an opening $5 \mathrm{~mm}$ from the tip was inserted into the eye in the holder so that the tip was located in the posterior chamber and the hole in anterior chamber, to prevent the deepening of anterior chamber.

The position of needle and eye in the holder was fixed in gypsum. The surface of eye was covered with wet paper to prevent dehydration. A valve was introduced between needle and tube to detach and reattach the eye to the outflow measurement setup for the laser treatment. The intraocular pressure (IOP) was controlled by adjusting the height difference between the surface of the liquid in reservoir and the tip of needle. The mass of liquid in reservoir was measured periodically by a micro scale connected electronically to a computer using Labview 7.1 (National Instruments).

Experimental procedures. Enucleated rabbit eyes no older than 48 hours postmortem were used in the studies. As the first step, aqueous outflow rate was established in an untreated eye. This step is referred to as baseline measurement and was conducted in the following order:

(1) The perfusion needle was inserted into the eye as the above described procedure and position. Then the eye and the needle were incased in gypsum.

(2) The flow was started by opening the valve in between the reservoir and the eye, and the flow rate was stabilized by leaving the eye setup intact for 30 minutes at $10 \mathrm{mmHg}$ perfusion pressure.

(3) The perfusion pressure, also the IOP in this setup, was set to $15 \mathrm{mmHg}$ by adjusting the height difference between the surface of solution in reservoir and the eye globe.

(4) The change of mass of the reservoir was measured every 5 seconds for 10 minutes by the micro scale connected to the computer. 


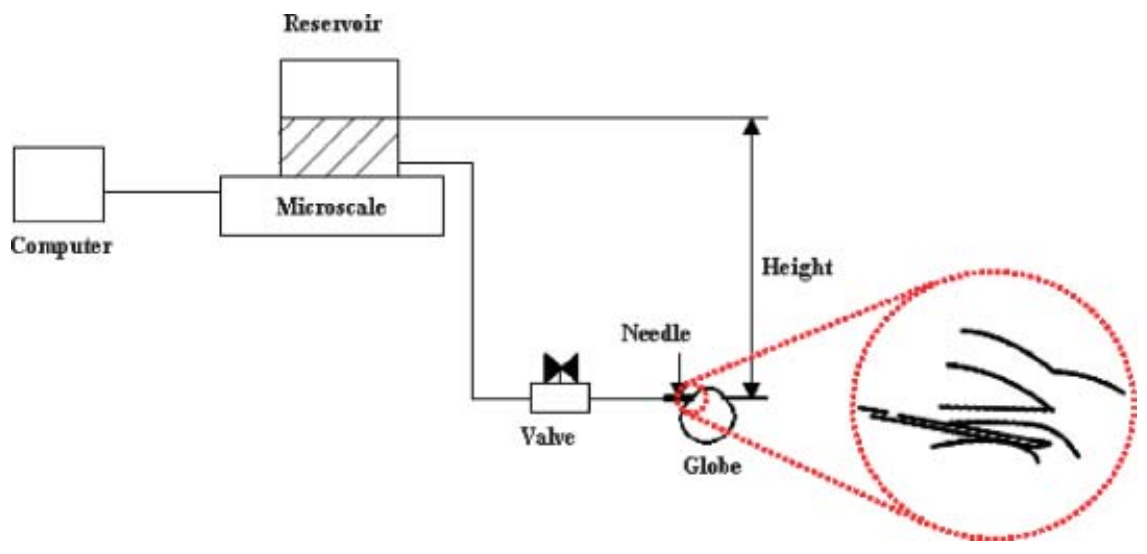

Fig. 7. Schematic of outflow measurement setup with inset showing details of needle insertion.

(5) Steps 3 and 4 were repeated at $25 \mathrm{mmHg}$ perfusion pressure.

Flow rates were measured in the unit of mass/time and converted into volume/time ( $\mu \mathrm{l} /$ minute) by taking into account of the density of the BSS solution in the reservoir. The valve was closed after the baseline measurement, and the eye and needle contained in the holder were detached from the outflow measurement setup.

A microscope slide was placed on the globe at the position where the channel needed to be created, and the eye was placed on the scanner stage. Laser beam energy was set to be $17 \mu \mathrm{J}$. To determine the zero depth (reference point for the scanning in the $Z$ direction), the focal point of laser beam was adjusted to the bottom surface of the applanation glass (anterior scleral surface). Before starting the scanning process the focal point of the laser was moved into sclera, so that the optical breakdown occurred at or below the posterior scleral surface. First, a raster pattern was scanned in the $X Y$ plane with spot and line separations of 3 and $5 \mu \mathrm{m}$, respectively. Then, the eye was moved downward by $2 \mu \mathrm{m}$, the layer separation, so that the focal point of the laser beam was moved toward the anterior surface and the raster scanning was repeated again in the $X Y$ plane. The scanning was stopped before the channel reached the anterior surface of the sclera so that channel was created with depth of approximately $90 \%$ of scleral thickness.

After finishing the creation of the partial thickness drainage channel, the eye was reconnected to the outflow measurement setup. The outflow rate was measured following the outflow measurement protocol described above. The flow rate was selected as the steady state outflow rate that kept same value for at least 2 minutes.

\section{RESULTS}

Figure 9 displays an OCT image of a partial thickness drainage channel created at the angle of the iris and cornea. The channel creation was stopped before the photodisruptions created by the femtosecond laser reached the anterior surface of the sclera. The border lines of the channel are highlighted by yellow lines in the figure.

Experimental data from three enucleated rabbit eyes were fit to the model. In the first eye, a partial thickness channel with dimensions, shown in Figure 8, of $800 \mu \mathrm{m}$ (width) $\times 320 \mu \mathrm{m}$ (length) was created with the femtosecond laser. In the second eye, channel dimensions were $800 \mu \mathrm{m}$ (width) $\times 240 \mu \mathrm{m}$ (length). In the third eye, channel dimensions were $800 \mu \mathrm{m}$ (width) $\times 180 \mu \mathrm{m}$ (length). In every case, the channel depth was approximately $90 \%$ of the scleral thickness. The channel depth was measured on a time domain OCT image after completing the laser treatment.

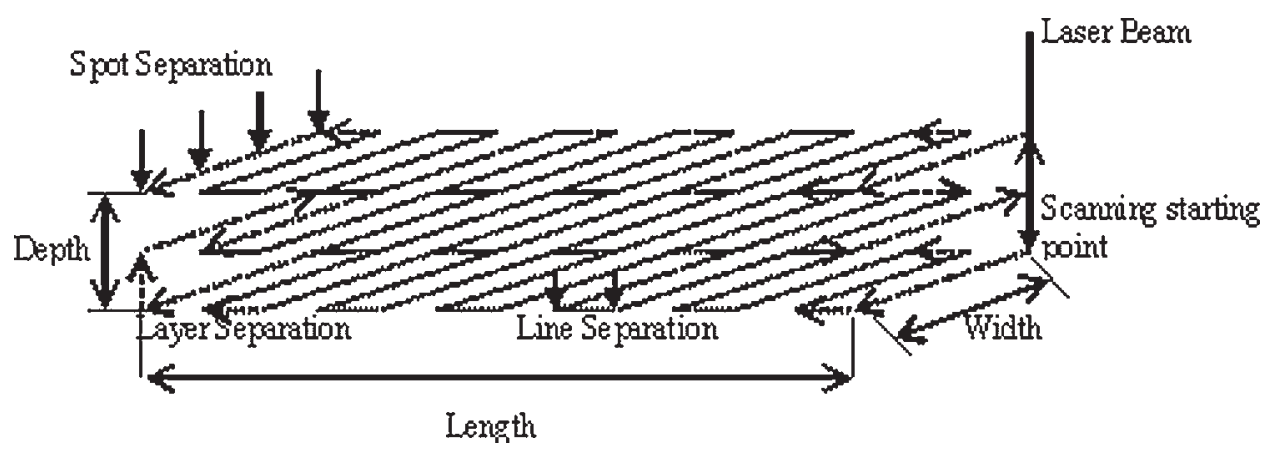

Fig. 8. Raster pattern for the channel creation. 


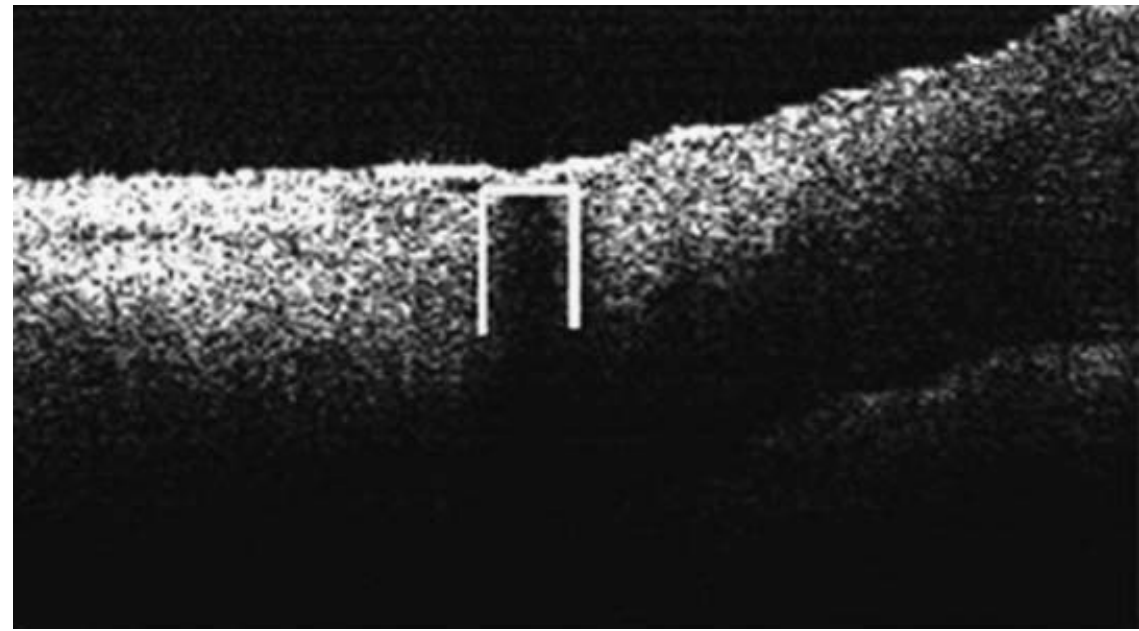

Fig. 9. Spectral domain OCT image of the subsurface scleral channel.

The results of $\mathrm{AH}$ outflow measurements before and after the laser treatment are provided in Figure 10. The value of the preoperative outflow rate varied from 1.3 to $2.0 \mu \mathrm{l} / \mathrm{minu}$ te at $15 \mathrm{mmHg}$ perfusion pressure and from 2 to $2.2 \mu \mathrm{l} /$ minute at $25 \mathrm{mmHg}$ perfusion pressure. The postoperative outflow rate ranged from 3.5 to $4.6 \mu \mathrm{l} /$ minute at $15 \mathrm{mmHg}$ perfusion pressure and from 5.9 to $12.5 \mu \mathrm{l} /$ minute at $25 \mathrm{mmHg}$ perfusion pressure. This increase is considerably larger than any washout effect measured in cadaver eye perfusion experiments in various species [19] and in vivo rabbit eye [20], indicating significantly increased outflow after partial thickness drainage channel creation by the femtosecond laser.

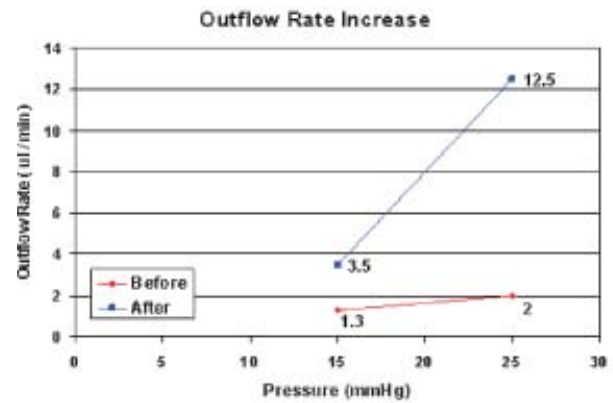

a Eye with $800 \mu \mathrm{m} \times 320 \mu \mathrm{m}$ channel
$\mathrm{AH}$ flow was simulated in $2 \mathrm{D}$ and $3 \mathrm{D}$ models and the result in $2 \mathrm{D}$ model is displayed in Figure 11. It can be seen in Figure 11 that AH entered the anterior chamber through the gap between the lens and the iris and flowed toward the cornea then exited the eye through the trabecular pathway. The calculated values of IOP from the $2 \mathrm{D}$ and $3 \mathrm{D}$ models are in agreement within $96 \%$. The red lines in Figure 12 display the $3 \mathrm{D}$ finite element simulation of the $\mathrm{AH}$ flow through the remaining sub-conjunctival layer at the anterior end of the partial thickness channel.

To simulate glaucoma conditions the permeability of the outflow pathway was determined from the measured outflow rate at $25 \mathrm{mmHg}$ (the normal value IOP in the

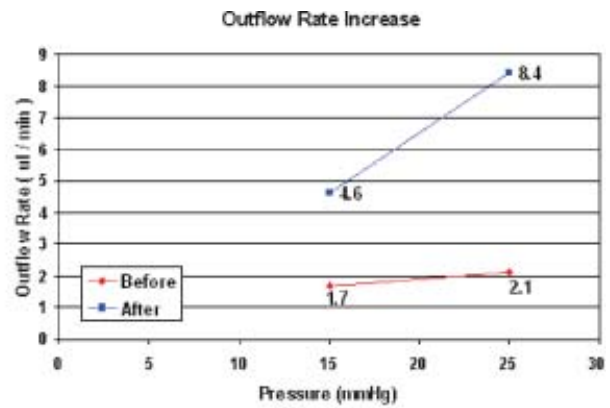

b Eye with $800 \mu \mathrm{m} \times 240 \mu \mathrm{m}$ channel

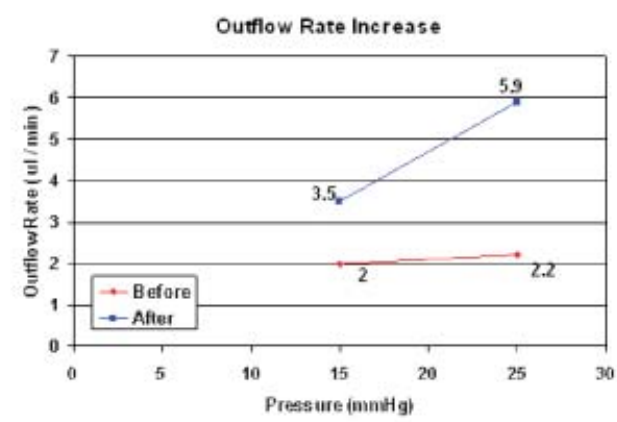

c Eye with $800 \mu \mathrm{m} \times 180 \mu \mathrm{m}$ channel

Fig. 10. Outflow rate as a function of IOP before and after laser treatment. 


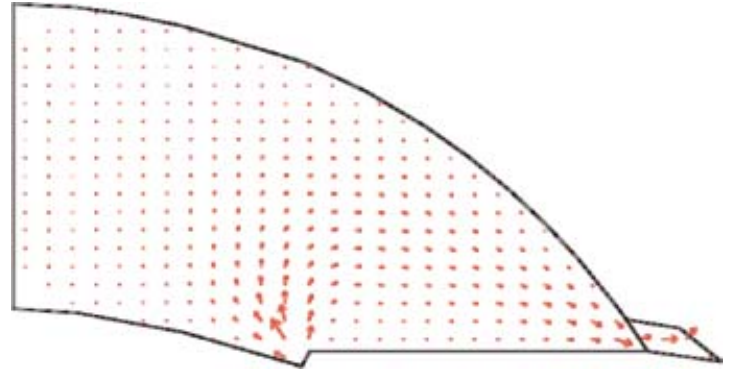

Fig. 11. Profile of the AH flow in the 2D model in the simplified geometry shown in Figure 2b. Color code represents velocity of $\mathrm{AH}$.

rabbit eye is $16.5 \mathrm{mmHg}$ [21]). In eye $\# 1$, the pathway permeability was calculated to be $4.56 \times 10^{-16} \mathrm{~m}^{2}$ by fitting data obtained in outflow measurements before the laser treatment to the 2D model. Entering this value of outflow pathway permeability and the outflow rate measured after the laser treatment into the 3D model, the effective channel permeability introduced in the mathematical model was determined to be $8.67 \times 10^{-15} \mathrm{~m}^{2}$ by varying its value until $25 \mathrm{mmHg}$ IOP was obtained. In eye \#2 and eye \#3, the pathway permeability was calculated and the effective channel permeability was determined to be $6.78 \times 10^{-15}$ and $5.45 \times 10^{-15} \mathrm{~m}^{2}$ in the same way as in eye \#1. Entering the mean effective channel permeability, $6.96 \times 10^{-15} \mathrm{~m}^{2}$, IOP was predicted to be $29.7 \mathrm{mmHg}$ in eye $\# 1,24.6 \mathrm{mmHg}$ in eye \#2, and $21.9 \mathrm{mmHg}$ in eye \# 3 .

To simulate the effect of the femtosecond laser created partial thickness channels on the IOP in more clinically realistic case, we closed the channels in our model while we kept the inflow rate at $25 \mathrm{mmHg}$ the same as before the creation of the channel. This model mimics the pathophysiology in a glaucoma eye, when the AH production rate is normal while the outflow is reduced. In this case

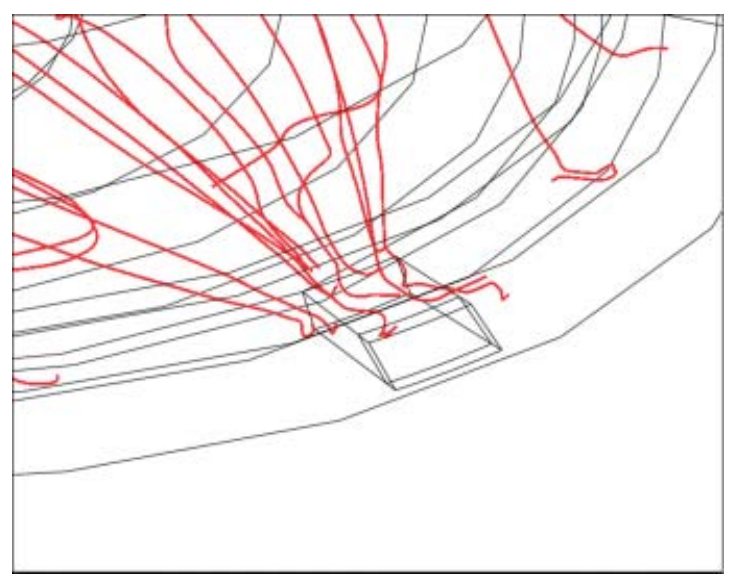

Fig. 12. The AH flow through the channel as shown by red line. Black lines are representing the same geometry shown in Figure 4. the model predicted a considerable decrease of the IOP for all three eyes; 4.7, 6.2, and $8.2 \mathrm{mmHg}$ in eyes \#1, \#2, and \#3, respectively. This simulation indicates that substantial IOP reductions can be achieved with the femtosecond laser created partial thickness channels.

\section{DISCUSSION}

The goal of this study was to develop a preliminary FEM to assist in the prediction of IOP reduction by partial thickness scleral drainage channels created with the femtosecond laser. While the 2D model can moderately describe flow dynamics in the intact eye, a more realistic 3D model is required for accurate modeling of the effect of the channel on IOP.

The lack of information on the dimension, position and permeability of each layer in AH outflow pathway introduced some challenge for this study. We had to assume that the permeability of the pathway can be represented with a comprehensive permeability value, not with the exact summation of the permeability of each layer. Although this assumption might introduce a considerable simplification to the model, it was satisfactory for comparison with the current experimental data, since the outflow rate was measured by the amount of liquid entered into the eye without measuring the pressure distribution through the pathway.

Modeling outflow in enucleated cadaver eyes provided another challenge for this study. Since there is no blood flow in the episcleral vein, the boundary condition for final destination of AH cannot be used in the model. To describe outflow in the enucleated rabbit eyes, we assumed that $\mathrm{AH}$ flows through the pathway to the surface of the eye, therefore boundary conditions were set to the atmospheric pressure rather than the episcleral vein pressure.

Uveoscleral flow was neglected in the model, because its exact flow rate is not known. This assumption may not result in considerable errors in the model as we focused on modeling the effect of the partial thickness drainage channels on the outflow dynamics. The partial thickness drainage channel was modeled by assigning appropriate permeability value for the remaining thin scleral layer at the anterior end of the channel. The thickness of the remaining layer of the sclera is expected to be the primary condition determining the amount of IOP reduction.

The validity of the model was checked by outflow measurements in cadaver rabbit eyes before and after channel creation with the femtosecond laser. The noninvasive measurement of the dimensions and position of the achieved channels was difficult since traditional light microscopy imaging does not work in the translucent sclera. Although ultrasonic imaging has adequate resolution for the observations of the channels, simultaneous coupling of the ultrasound and the laser beam into the tissue may be difficult, and the acoustic coupling material may interfere with the perfusion measurements. Therefore, noninvasive time domain OCT imaging was chosen to check the shape and position of the channel. Additionally, scleral thickness was measured after the experiments and found to range from 0.6 to $0.8 \mathrm{~mm}$ in the three eyes. 
Slightly different scleral thicknesses and different channel dimensions may partially account for the range in the measured values of $\mathrm{AH}$ outflows shown in Figure 10.

Our experimental observations of the outflow before and after the channel creation are within acceptable range of the model predictions. Therefore, we conclude that none of the simplifying assumptions led to serious errors in the model. On the other hand, the model discussed in this paper is still in its early development and requires additional refinement, and also comparison with in vivo data.

In summary, this study demonstrates the potential of a 3D FEM to describe aqueous outflow dynamics and predict IOP reduction by femtosecond laser created partial thickness scleral channels. Although the model provided reasonable agreement with experimental observations in enucleated rabbit eye, further enhancement of the model is necessary for more accurate predictions. The ultimate goal of the project is to apply the model to in vivo eyes. In that case, effects such as heat transfer from cornea and iris will also be integrated into the model.

\section{ACKNOWLEDGMENTS}

This research is supported in part by the National Institutes of Health (NIH Grant: R01 EY014456-02). The authors also with thank Professor Zhongping Chen for providing the OCT device for the experiments and Dr. Ron Kurtz for valuable discussions.

\section{REFERENCES}

1. Distelhorst JS, Hughes GM. Open-angle glaucoma. Am Family Physician 2003;67(9):1937-1944.

2. Morrison JC, Pollack IP. Glaucoma: Science and Practice. New York: Thieme Medical Publishers; 2003.

3. Berlien HP, Muller GJ, editors. Applied Laser Medicine. Berlin, New York: Springer; 2003.

4. Juhasz T, Kastis GA, Suarez C, Bor Z, Bron WE. Timeresolved observations of shock waves and cavitation bubbles generated by femtosecond laser pulses in corneal tissue and water. Lasers Surg Med 1996;19(1):23-31.
5. Kautek W, Mitterer S, Krüger J, Husinsky W, Grabner G. Femtosecond-pulse laser ablation of human corneas. Appl Phys A-Mater Sci Process 1994;58(5):513-518.

6. Du D, Squier J, Kurtz R, Elner V, Liu X, Guttmann G, Mourou G. Damage threshold as a function of pulse duration in biological tissue. In: Barbara PF, Know WH, Mourou G, Zewail JH, editors. Ultrafast phenomena IX. New York: Springer-Verlag; 1994. pp 254-255.

7. Sacks ZS, Kurtz RM, Juhasz T, Mourau GA. High precision subsurface photodisruption in human sclera. J Biomed Opt 2002;7(3):442-450.

8. Juhasz T. The effect of femtosecond laser scleral treatments on the outflow of aqueous humor. Invest Ophthalmol Vis Sci 2004;45: U392 Suppl 1.

9. Huang EC, Barocas VH. Accommodative microfluctuations and iris contour. J Vis 2006;6(5):653-660.

10. Kumar S, Acharva S, Beuerman R, Palkama A. Numerical solution of ocular fluid dynamics in a rabbit eye: Parametric effects. Ann Biomed Eng 2006;34(3):530-544.

11. Barocas VH, Heys JJ. Computer simulation of passive iris deformation during blinking and accommodation. Invest Ophthalmol Vis Sci 2001;42(4): 3563 Suppl S.

12. Chai D, Chaudhary G, Kurtz RM, Juhasz T. Aqueous humor outflow effects of partial thickness channel created by a femtosecond laser in ex vivo human eyes. Proc SPIE 2007; 6435:643500-1.

13. Prince JH, Harvey JH, editors. The rabbit in eye research. Springfield, IL: Charles C. Thomas; 1964

14. Silver DM, Quigley HA. Aqueous flow through iris-lens channel: Estimates of differential pressure between the anterior can posterior chambers. J Glaucoma 2004;13(2): 100-107.

15. Sokol J, Stegman Z, Liebmann JM, Ritch R. Location of the iris insertion in pigment dispersion syndrome. Ophthalomology 1996;103(2):289-293.

16. Reitsamer HA, Kiel JW. A rabbit model to study orbital venous pressure, intraocular pressure, and ocular hemodynamics simultaneously. Invest Opthalmology \& Visual Science; 2002;43(12):3728-3734.

17. Brubaker RF. The effect of intraocular pressure on conventional outflow resistance in the enucleated human eye. Invest Ophthalmol Vis Sci 1975;14(4):286-292.

18. Erickson-Lamy K, Schroeder AM, Bassett-Chu S, Epstein DL. Absence of time-dependent facility increase (washout) in the perfused enucleated human eye. Invest Ophthalmol Vis Sci 1990;31(11):2384-2388.

19. Ruben JB, Moses RA, Grodzki WJ. Perfusion outflow facility in the rabbit eye. Invest Ophthalmol Vis Sci 1985;26(2):153158.

20. Lu DW, Chang CJ, Wu JN. The change of vitreous $\mathrm{PH}$ values in an acute glaucoma rabbit model. J Ocul Pharmacol Ther 2001;17(4):343-350. 